\title{
Fiber Laser Cutting of Alumina Substrates for Electronic Applications Comparison of the Experimentally Determined and Simulated Influence on the Mechanical Strength
}

\author{
Benedikt Adelmann and Ralf Hellmann \\ Applied Laser and Photonics Group, University of Applied Sciences Aschaffenburg, Wuerzburger Strasse 45, Aschaffenburg 63743, \\ Germany
}

\begin{abstract}
This study compares experimentally determined and simulated mechanical strength of different geometries laser cut into alumina ceramic substrates. A digital power modulated $500 \mathrm{~W}$ single mode fiber laser is used to cut differently shaped inner contours into rectangular ceramic substrates. The flexural strength of these samples is measured by a 3-point-bending test. In addition, employing a finite element simulation the principal stress of these geometries during a 3-point-bending is simulated with a linear elastic model. The reciprocal of the simulated stress is compared with the measured strength revealing an excellent agreement. We, therefore, can conclude that the strength reduction by cutting inner contours can be exclusively explained by the geometry of the rectangular, which in turn implies that the fiber laser cutting process itself causes no relevant additional structural damage. These results highlight the superior quality of digital power modulated fiber laser cutting of ceramics. Moreover, we demonstrate by a further comparison between experimental and simulated results that an increased strength is feasible by displacing fragile parts of inner contours to areas with less mechanical load.
\end{abstract}

Key words: Laser cutting, fiber laser, flexural strength, alumina, strength simulation.

\section{Introduction}

During the last decade the demand for alumina ceramic substrates for electronic applications has increased significantly. Due to their manifold advantages over other substrate materials, such as paper and glass fiber reinforced plastics, ceramic substrates are found in, e.g., automotive, telecommunication, and power electronics as well as in various sensor systems. The most important of such advantages is the high thermal conductivity of electronic ceramics. Standard and frequently used FR4 has a thermal conductivity of $0.3 \mathrm{~W} / \mathrm{mK}$, while alumina is characterized by $25 \mathrm{~W} / \mathrm{mK}$ [1]. As a result, the need of additional cooling is reduced when using

Corresponding author: Benedikt Adelmann, M. Eng, scientific engineer, research field: laser material processing. ceramic substrates. Further advantages of alumina as compared to standard FR4 are the chemical inertness, lower dielectric loss factor and the much higher maximum operation temperature [2]. For interlayer connection, fixation and for through hole devices inner contours are necessary. Producing these inner contours requires efficient methods which do not damage the ceramic substrate even when cutting complex contours.

Due to its high hardness and chemical inertness alumina is very difficult to machine mechanically or chemically [3, 4]. Therefore, different laser sources and processing strategies have been investigated over the last two decades. Yet in industry, processing nowadays predominantly relies on $\mathrm{CO}_{2}$ lasers. However, laser machining is a thermal process resulting in mechanical stress [5]. This stress can reduce the mechanical strength of the material 

Experimentally Determined and Simulated Influence on the Mechanical Strength

significantly. Zhang et al. [6] cut rectangles in $3 \mathrm{~mm}$ thick silicon nitride composite ceramic with a pulsed $120 \mathrm{~W}$ Nd:YAG laser and measured the flexural strength after cutting with a 3-point-bending test. The results reveal a $60 \%$ strength reduction caused by the laser cutting process. Compared to that fiber laser cutting of $0.63 \mathrm{~mm}$ thick alumina substrate resulted in only 3\% strength reduction [7]. The importance of mechanical damage is further highlighted by a prominent number of publications about the creating of cracks during ceramic cutting.

Hong et al. [8] separated silicon nitride ceramics with a $250 \mathrm{~W}$ Q-switched $\mathrm{CO}_{2}$ laser in a multi-pass process with an effective cutting velocity of $1 \mathrm{~mm} / \mathrm{s}$. In this process less crack formation is achieved at higher velocities of the single passes of about $220 \mathrm{~mm} / \mathrm{s}$. Ji et al. [9] cut $10 \mathrm{~mm}$ thick alumina using a pulsed $\mathrm{CO}_{2}$ laser with $3.5 \mathrm{~kW}$ peak power at $0.3 \mathrm{~mm} / \mathrm{s}$ emphasizing the absence of substrate breakup.

To predict cracks induced by the thermal stress during laser cutting, Lee and Ahn [10] developed a Bayesian Probabilistic Model suggesting that cracks mainly depend on the cutting front angle. Another approach to predict cracks was presented by Yilbas et al. [5] using thermal simulations showing that cracks are mainly caused by the re-solidification of molten material in the cut, kerf. Yan et al. [11] performed experiments cutting $6 \mathrm{~mm}$ thick alumina with a $3.5 \mathrm{~kW}$ $\mathrm{CO}_{2}$ laser at different pulse modes showing that fewer pulses with more energy but large switch off times create less stress than many pulses with lower pulse energy.

To avoid cracks in ceramics processing short pulsed laser or excimer lasers have also been investigated. Wang et al. [12] presented results on cutting holes in $0.63 \mathrm{~mm}$ thick alumina substrates using a 150 femtosecond short pulse laser with an average power of $0.8 \mathrm{~W}$ in a multi pass process. The authors achieved very high cutting quality but only an effective cutting velocity of $1.5 \mu \mathrm{m} / \mathrm{s}$, which is much too slow for industrial applications. The use of a $\mathrm{KrF}$ excimer laser at $248 \mathrm{~nm}$ by Lump and Allen [13] in cutting vias into $0.63 \mathrm{~mm}$ thick alumina nitride showed good quality but revealed major problems with cracks. With 3,000 pulses for one via at $50 \mathrm{~Hz}$ laser repetition rate the process is also too slow for industrial applications.

In ceramics the cut geometry has a major influence on the crack formation. Black and Chua [14] cut complex contours in $9.2 \mathrm{~mm}$ thick alumina with a power modulated $530 \mathrm{~W} \mathrm{CO}_{2}$ laser at $1.5 \mathrm{~mm} / \mathrm{s}$ and report significant problems with cracks inside and near sharp corners. In addition, inner geometries result in a significant lower strength of the ceramic during fiber laser cutting [15].

To recapitulate, literature reveals that laser cutting of ceramics often results in cracks in the substrate and a corresponding reduction of the strength. Especially, complex contour cutting leads to major crack formation which in turn results in a weakening of the material. Hence, it is essentially necessary to determine whether the observed strength reduction during contour cutting in ceramic is caused by the impacts of the laser process or by the chosen geometry. We, therefore, compare the measured strength of different geometries cut in alumina with simulated stress results for these geometries using COMSOL Multiphysics ${ }^{\circledR}$ Structural Mechanics Module. As we have recently shown that fiber lasers can be employed to efficiently cut alumina [15] despite the unfavorable emission wavelength in the near infrared as compared to frequently used $\mathrm{CO}_{2}$ lasers, this comparative study is continuatively also based on a digitally modulated fiber laser.

\section{Experimental setup}

\subsection{Laser System}

In this study, a continuous wave $500 \mathrm{~W}$ single mode fiber laser (IPG Photonics) was used to perform the experiments. The laser system is equipped with linear stages (x, y) for the workpiece (Aerotech) and a fine cutting head (Precitec) attached to a linear drive ( $\mathrm{z}$ ). The assisted gas (nitrogen) with purity greater than 99.999\% flows coaxial to the laser beam. The gas 
nozzle has a diameter of $0.3 \mathrm{~mm}$ and its distance to the workpiece is positioned by the z-linear drive. The emission wavelength of the laser is specified to be $1,070 \mathrm{~nm}$ in conjunction with a beam propagation factor of $\mathrm{M}^{2}<1.1$. The raw beam diameter of $7.25 \mathrm{~mm}$ is focused by a lens with focal length of $50 \mathrm{~mm}$. The resulting Rayleigh length is calculated to $70 \mu \mathrm{m}$ and the focus beam diameter $\left(\mathrm{e}^{-2}\right)$ is $11 \mu \mathrm{m}$. The laser system is equipped with a PSO (position synchronous output) to modulate the laser digitally. The maximum digital modulation frequency for the laser is $50 \mathrm{kHz}$. Using the PSO, the laser is switched on for an adjustable time after the drives moved a given distance. With the PSO the energy input per unit length is kept constant during acceleration and deceleration paths (i.e. during cutting a corner).

In order to minimize the thermal impact during laser cutting on the ceramic material, the laser is digitally modulated using the PSO. The maximum laser power of $500 \mathrm{~W}$ is switched on for a period of $1 \mathrm{~ms}$ after the drives travelled a distance of $10 \mu \mathrm{m}$. The travel distance is chosen with respect to the focus diameter of $11 \mu \mathrm{m}$. Using a velocity of $1 \mathrm{~mm} / \mathrm{s}$ results in a duty cycle of $10 \%$. Further parameters are a gas pressure of $6 \mathrm{bar}, 0.3 \mathrm{~mm}$ nozzle diameter and a focus position on the surface of the material.

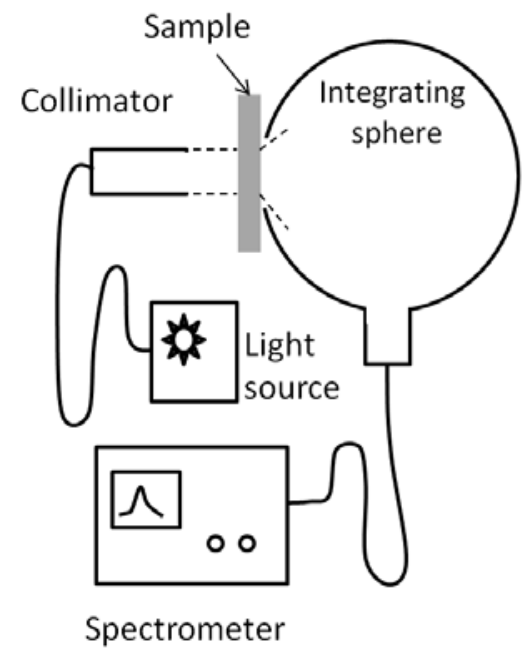

\subsection{Alumina Ceramic}

The substrate material used in the experiments is alumina (CeramTec) with a thickness of $0.63 \mathrm{~mm}$, one of the most commonly used thickness in electronic industry. The material has a purity of more than $96 \%$ and a density of $3.5 \mathrm{~g} / \mathrm{cm}^{3}$. For laser processing the optical properties such as reflectivity and absorption of alumina are important. Therefore, the transmission and reflection spectra of alumina are measured with a broad band light source, an integrating sphere and an optical spectrometer at room temperature. As depicted in Fig. 1 for the transmission measurement a broad band light source illuminates the sample with the transmitted radiation being collected with an integrating sphere and being guided to a fiber coupled optical spectrometer. For reflection measurements, the light source radiates through an aperture into the integrating sphere onto the sample and the reflected radiation is collected by the integrating sphere and guided to the spectrometer.

The percentage of reflected and transmitted radiation is shown in Fig. 2. It is obvious that alumina has an almost constant reflection and transmission between $400 \mathrm{~nm}$ and 1,200 nm (visible and near infrared region). In detail the optical reflection is determined to $81 \%$ at $1,070 \mathrm{~nm}$ wavelength, i.e., the emission wavelength of the applied fiber laser. This is comparable to the reflectivity

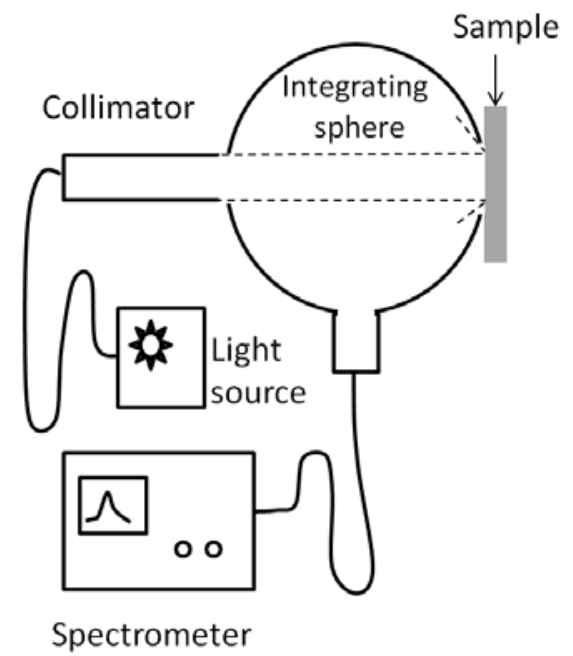

Fig. 1 Schematic design for the transmission (left) and reflection measurement (right). 


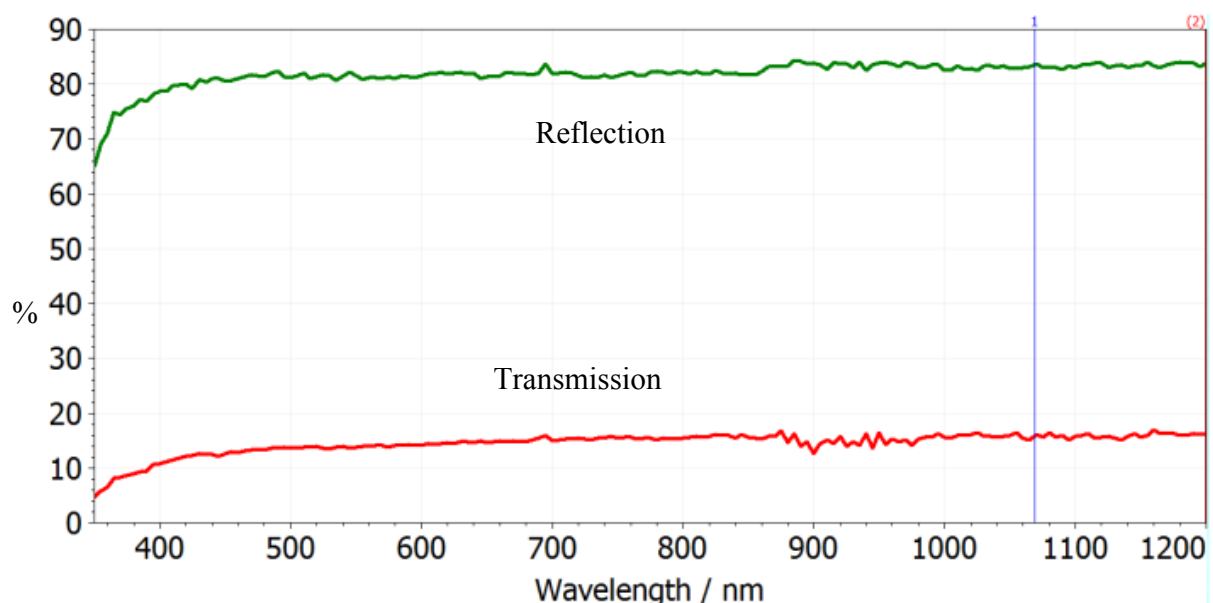

Fig. 2 Reflection and transmission spectrum of $\mathrm{Al}_{2} \mathrm{O}_{3}$ with $0.63 \mathrm{~mm}$ thickness, the vertical bar illustrates the laser wavelength.

of $79 \%$ reported by Liu et al. [16]. With respect to the laser cutting process using a near infrared fiber laser, please note that Zhang and Modest [17] published experimental results showing that the absorption of alumina at 1,064 nm significantly increases to more than $90 \%$ at temperatures exceeding the melting point of the material. The room temperature transmission is determined to $15 \%$ at $1,070 \mathrm{~nm}$. Please note that the reflected and transmitted radiation for these ceramic substrates is strongly scattered rather than directed.

\subsection{Flexural Strength Measurement}

To measure the flexural strength, a 3-point-bending test machine (Thuemler) is used with $20 \mathrm{~mm}$ distance between the two contact points. Fig. 3 shows the schematic diagram of the 3-point-bending test including the relevant dimensions.

The flexural strength $\sigma_{\text {flex }}$ is calculated with Eq. (1) from the maximum force $F$ which the sample withstands before breaking. Here $l$ is the distance between the two contact points, $b$ stands for the width of the sample and $d$ expresses the sample thickness. The velocity of the bending test is set to $1 \mathrm{~mm} / \mathrm{min}$ as recommended by Bengisu [2].

$$
\sigma_{f l e x}=\frac{3 \cdot \mathbf{F} \cdot \mathbf{l}}{2 \cdot \mathbf{b} \cdot \mathbf{d}^{2}}
$$

To assess the influence of laser cut inner contours on the flexural strength, four different inner contours (namely a circle, 2 circles, a rectangle and a rhombus) are cut into the middle of the samples as shown in Fig. 4. The width of all rectangular samples is $4 \mathrm{~mm}$ and the inner contours reduce the width in the center of the rectangular by $1 \mathrm{~mm}$. For the calculation of the flexural strength the effective width of $3 \mathrm{~mm}$ is used. For reference purposes, additionally $3 \mathrm{~mm}$ wide rectangles without inner contours are cut and the flexural strength measured.

\subsection{Simulation}

To evaluate whether the observed strength reduction is caused by the impacts of the laser process or the chosen geometry itself, we simulated the 3-point bending test using COMSOL Multiphysics ${ }^{\circledR}$ Structural Mechanics Module. In the stationary model, the geometries from Fig. 4 and the reference sample (rectangular without any inner contour) are designed as cut on the laser machine (width of $22 \mathrm{~mm}$ and a thickness of $0.63 \mathrm{~mm}$ ). Because alumina is a brittle ceramic which is not able to deform plastically, the samples are treated as a linear elastic material following Hooke's law (Eq. (2)). In this equation $\sigma$ represents the mechanical stress, $\boldsymbol{E}$ the Young's modulus and $\varepsilon$ the strain.

$$
\sigma=\boldsymbol{E} \cdot \varepsilon
$$

For the bending test two bars with $20 \mathrm{~mm}$ distance are built under the sample. The bars are not fixed to the sample in order to allow a horizontal movement of the sample. In addition, inside the sample no initial forces 


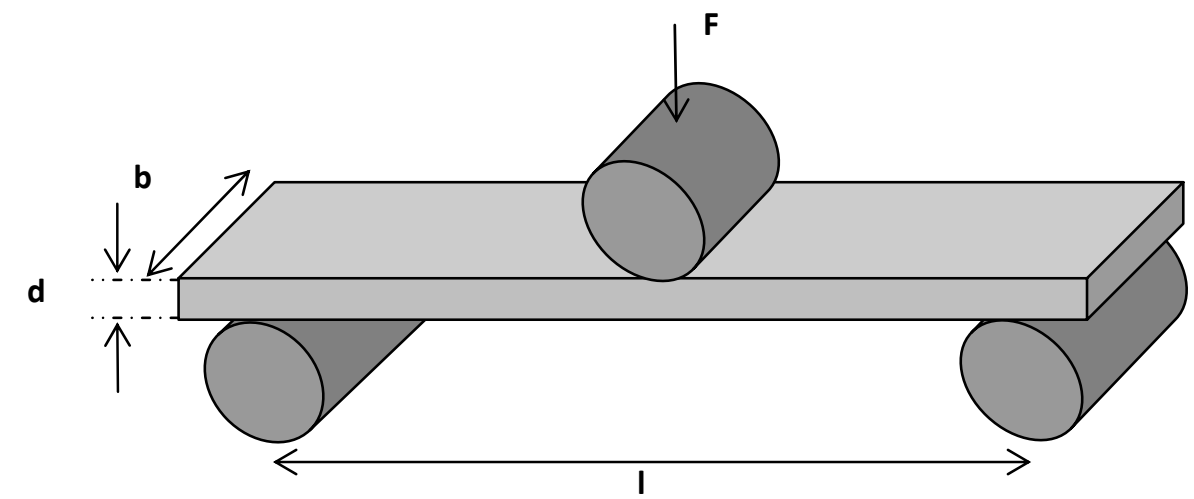

Fig. 3 Schematic diagram of the 3-point-bending test.
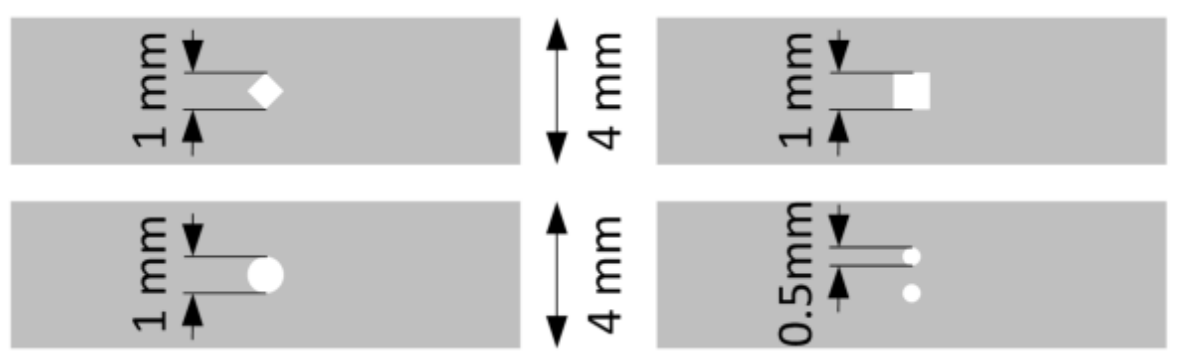

Fig. 4 Geometries of the specimen with different inner contours.

are applied. In the middle of the specimen a line force with $10 \mathrm{~N}$ is applied on the entire width of the sample. This magnitude of this force is chosen with respect to the typical breakup forces of the ceramic samples.

The mechanical stress in 3 dimensional materials is described by the stress tensor $\boldsymbol{S}$ in according Eq. (3) containing the normal stresses $\sigma_{x, y, z}$ in all spatial directions on the diagonal and the shear stresses $\tau$ in two spatial directions perpendicular to each normal stress (elements outside the diagonal).

$$
\boldsymbol{S}=\left(\begin{array}{ccc}
\sigma_{x} & \tau_{y x} & \tau_{z x} \\
\tau_{x y} & \sigma_{y} & \tau_{z y} \\
\tau_{x z} & \tau_{y z} & \sigma_{z}
\end{array}\right)
$$

The eigenvalues of the stress tensor calculated with Eq. (4) using the unit matrix $I$ is the principal stress $\sigma_{\mathrm{Hi}}$ [18]. Geometrically, this effect can be explained by rotating the volume element of the stress tensor in order to eliminate the shear strength.

$$
\operatorname{det}\left(\boldsymbol{S}-\sigma_{H i} \times \boldsymbol{I}\right)=0
$$

Because of its brittleness alumina breaks up at once when the maximum of the principal stresses exceeds the material strength [19]. Therefore, the maximum principal stress is calculated to locate the area in the sample which in practice would initiate the mechanical breakup.

\section{Results}

\subsection{Strength for Different Inner Contours}

The series of samples with the geometries shown in Fig. 4 and the reference sample are cut with the fiber laser (parameters as mentioned in section 0 ). The flexural strength of the cut specimen is measured with the 3-point-bending test and the results as well as their standard deviation are shown in Fig. 5. It is obvious that the reference sample has the highest strength follow by the circle. The lowest strength is measured by samples with edges in the inner geometry.

The same geometries are modeled during 3-point-bending with a force of $10 \mathrm{~N}$ and the principal stresses in the samples are calculated. The spatial distribution of the stress at the bottom side of the samples 


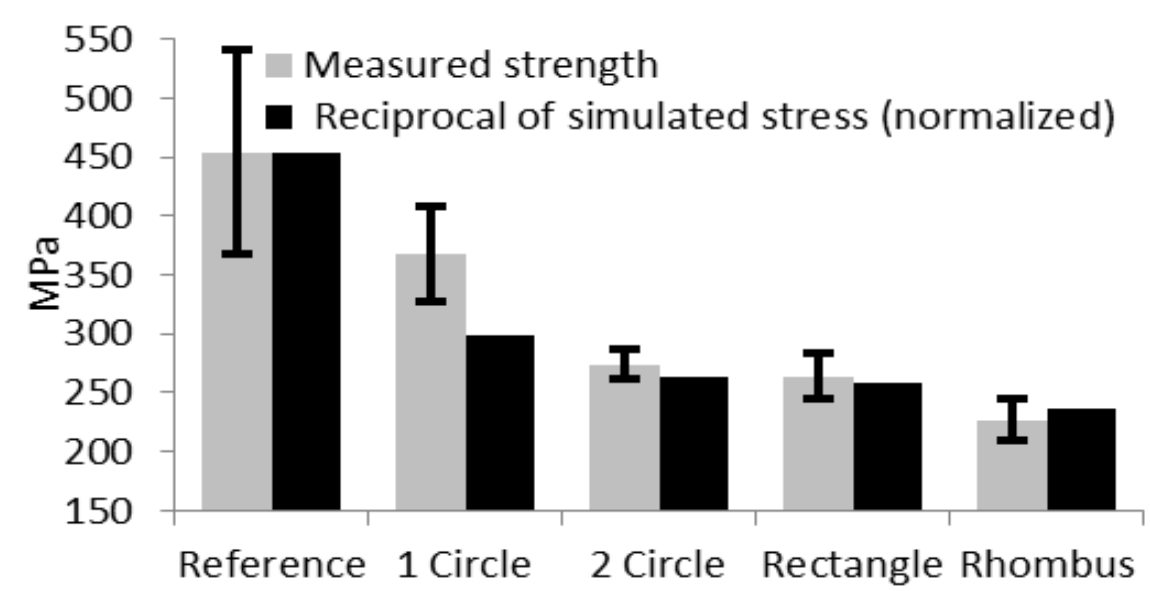

Fig. 5 Comparison between measured strength and the reciprocal of the normalized stress of samples with different inner contours.

is illustrated in Fig. 6, with red colors indicating a high and blue a low mechanical stress. Here, the bottom side of the samples is shown as on this side the impact of the bending test is higher as compared to the top side.

Fig. 6 reveals the highest stress in the middle of the sample at the edges of the inner contours. Please note that the stress is normalized to the maximum stress of each sample. For the sample with an inner circle (Fig. $6 a)$, the area of high stress is bigger and the forces are further spread which, in turn, results in a lower maximum stress. For the samples including the rhombus and the rectangle (Figs. $6 \mathrm{c}$ and $6 \mathrm{~d}$ ), the stress is concentrated at the corners of the inner contour which leads to a much higher maximum stress.

The area with the maximum stress initiates the breakup [19] which is experimentally confirmed during the bending test for all types of inner contour: the specimens with inner circles break up through the middle of the circles and the sample with the rhombus breaks up exactly through the 2 edges with the highest simulated stress. Accordingly, the samples with a rectangle inner contour, always break up at two adjoined edges rather than in the middle of the inner rectangle.

A high maximum stress at constant load means that only a low load can be applied before the maximum stress exceeds the critical stress of mechanical breakup. On the other hand, a low maximum stress at constant load results in a high possible load before the maximum stress reaches the critical stress of breakup [19]. Because the material is linear elastic, the reciprocal of the maximum stress is a sign of the mechanical strength. To obtain a comparable value to the measured strength, the reciprocal of the maximum simulated stress is normalized to the strength of the reference sample (454 MPa). These comparable values for the chosen geometries are depicted in Fig. 5. Apparently, the simulated results reveal by trend an excellent agreement with the experimental data and verify the tendency of lower strength for inner contours, especially those with edges. Therefore, we conclude that the strength reduction by cutting inner contours can be entirely explained by the geometry, i.e., the digitally modulated fiber laser cutting process causes no supplementary strength reduction. Additional damages in laser cut corners as reported, e.g., by Black and Chua [14] are not observed during modulated fiber laser cutting. In turn, these results highlight the superior quality of digital power modulated fiber laser cutting of ceramics for complex contours.

\subsection{Edge Displacement for Higher Strength}

In order to achieve a high strength even in cases when inner contours with edges are required, an improvement of the strength can be accomplished by a displacement of the weakening contours to areas with 
Fiber Laser Cutting of Alumina Substrates for Electronic Applications Comparison of the Experimentally Determined and Simulated Influence on the Mechanical Strength

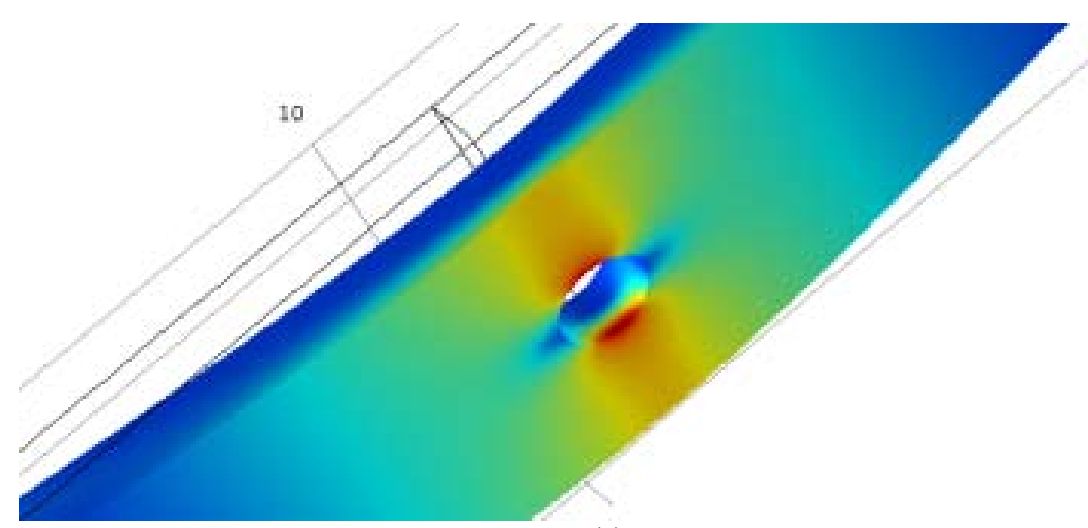

(a)

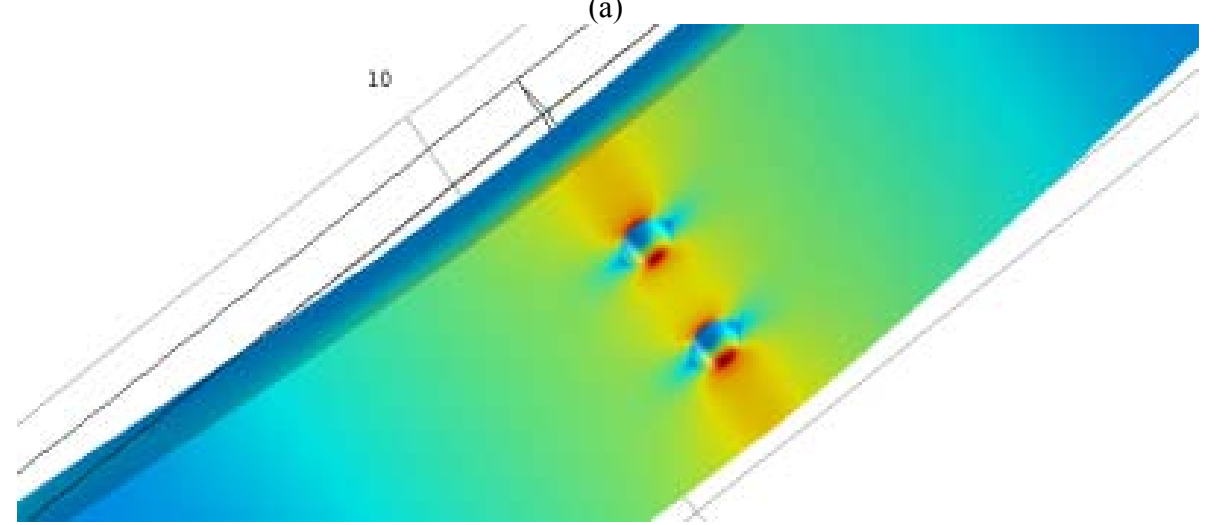

(b)

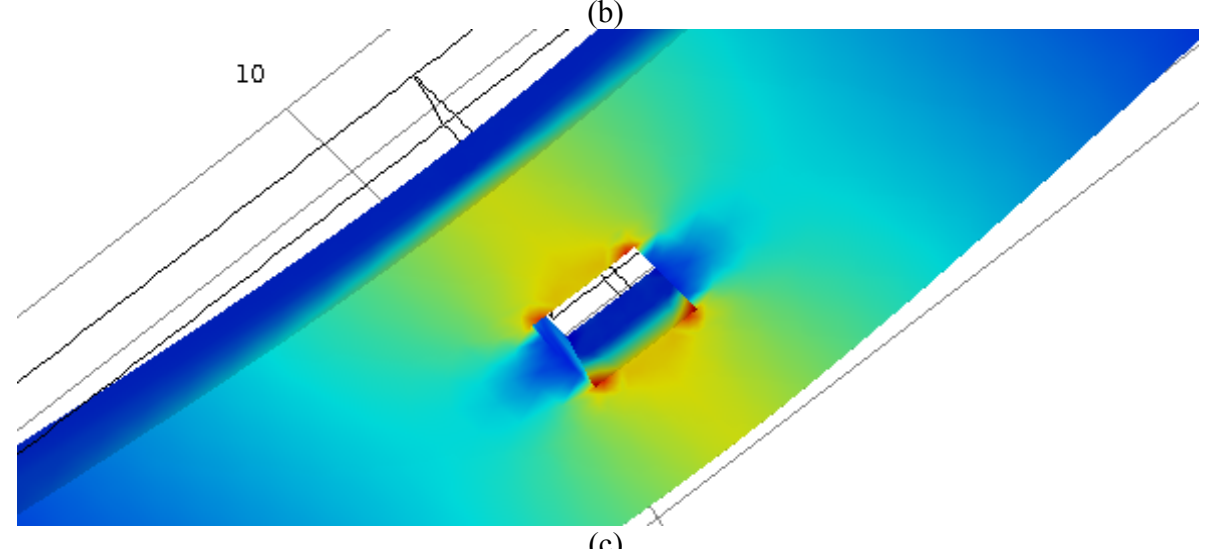

(c)

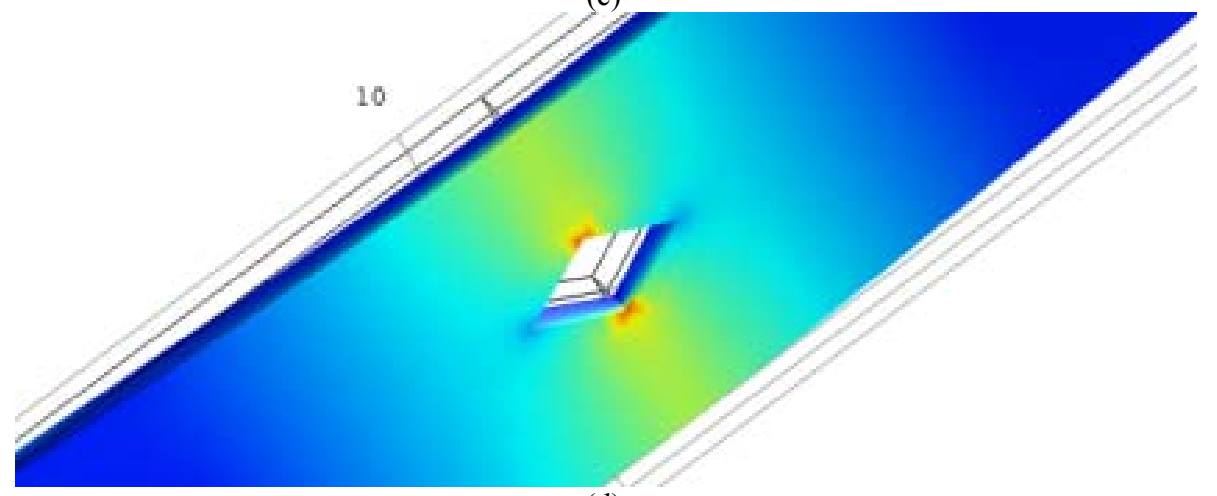

(d)

Fig. 6 Calculated normalized principal stress at the bottom of samples (middle between the bars) during 3-point-bending with $10 \mathrm{~N}$ with samples including inner contours: (a) circle; (b) 2 circles; (c) rectangle; and (d) rhombus. Red areas indicate a high stress whereas blue indicates a low stress. 


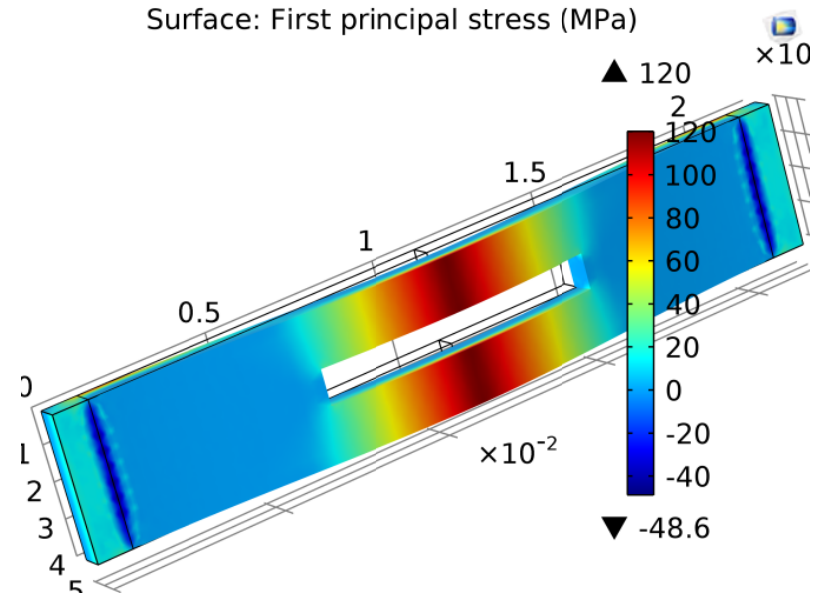

Fig. 7 Principal stress on the bottom side of a sample with 8 mm wide inner rectangle during 3-point-bending.

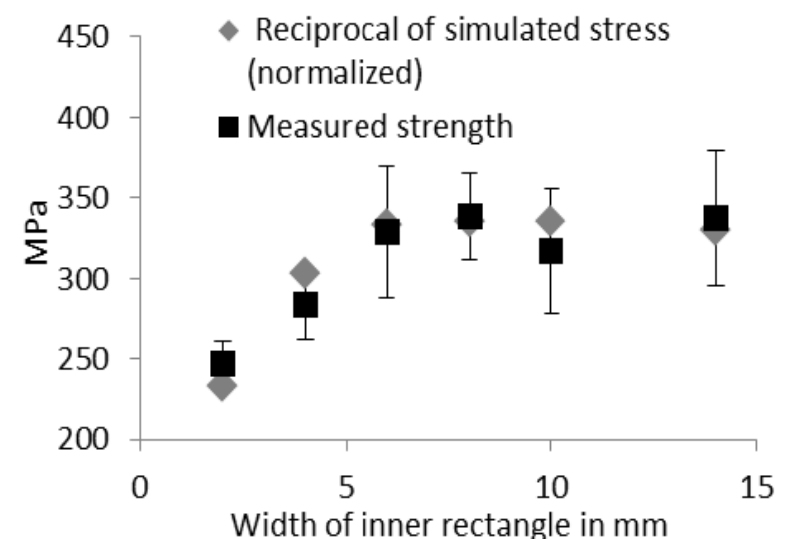

Fig. 8 Comparison between measured strength and the reciprocal of the normalized stress of samples with different wider inner rectangles.

lower load. During the 3-point-bending test the highest stress occurs in the middle of the sample with the stress decreasing in direction towards the bearings. For a sample with an inner rectangle, a higher strength can potentially be achieved by widening the inner rectangle in direction to the bearings. Fig. 7 reveals the simulated principal stress for such an expanded sample with an 8 $\mathrm{mm}$ wide inner rectangle during 3-point-bending test. Compared to Fig. 6c, in this case the maximum stress is located in the middle rather than at the edges of the inner rectangle. Furthermore, the area of maximum stress is significantly larger, i.e., the stress is spatially less concentrated. As a result, this sample should have a higher bending strength.

Fig. 8 shows the measured stress and the reciprocal simulated stress of samples with differently expanded inner rectangles. The simulated and measured data show by trend good agreement. At small inner rectangles the strength is significantly lower because the fragile edges of the rectangles are in the area of high load and therefore initiate the mechanical breakup which is also observed in the experiments. For $6 \mathrm{~mm}$ and wider inner rectangles the breakup is initiated in the middle of the sample where no edge is located. As a result, the strength does not vary for inner rectangles between $6 \mathrm{~mm}$ and $14 \mathrm{~mm}$ which can clearly be seen in Fig. 8. These results reveal that a displacement of weakening contours can increase the ceramic strength. From a general perspective, these results approve the excellent calculability of the strength variations for laser cut ceramics using the presented simulation approach.

\section{Conclusions}

We presented a comparative study of experimentally determined and simulated mechanical strength of different inner geometries cut into rectangular alumina ceramic substrates using a $500 \mathrm{~W}$ single mode fiber laser with digital power modulation at a velocity of 1 $\mathrm{mm} / \mathrm{s}$. The inner contours generated are one or two circles, a secondary rectangular and a rhombus. The flexural strength of these exemplarily chosen geometries is both measured by a 3-point-bending test and modeled employing a linear elastic model providing the principal stress during bending. The reciprocal of the principal stress is normalized to the strength of a reference sample and compared with the measured results of the flexural strength, which reveals excellent agreement. The results substantiate that the strength changes can be explained completely by the geometry and, as a consequence, we conclude that the digitally modulated fiber laser cutting process causes no supplementary strength reduction. These results also imply that the displacement of fragile corners out of the area with high mechanical load is a possible way to achieve higher strength for samples with inner 

Experimentally Determined and Simulated Influence on the Mechanical Strength

contour, which, in turn, is confirmed by both experimental and simulated results.

\section{References}

[1] Information Center Technical Ceramic. 2013. "Typial Properties of Technical Ceramics." [Online]. Available at: www.keramverband.de.

[2] Bengisu, M. 2001. Engineering Ceramics. Berlin: Springer.

[3] Samant, A. N., and Dahotre, N. B. 2009. "Laser Machining of Structural Ceramics-A Review." Journal of the European Ceramic Society 29: 969-93.

[4] Li, K., and Sheng, P. 1995. "Plane Stress Model for Fracture of Caramics during Laser Cutting." International Jorunal of Machine Tools \& Manufacture 35 (11): 1493-506.

[5] Yilbas, S. A., and Karatas, C. 2012. "Laser Straight Cutting Of Alumina Tiles: Thermal Stress Analysis." International Journal of Advanced Manufacturing Technology 58: 1019-30.

[6] Zhang, J., Lee, T., Ai, X., and Lau, W. 1994. "Investigation of the Surface Integrity of Laser-Cut Ceramic.” Journal of Materials Processing Technology 57: 304-10.

[7] Adelmann, B., and Hellmann, R. 2013. "Investigation on Flexural Strength during Fiber Laser Cutting of Alumina." Physics Procedia 41: 398-400.

[8] Hong, L., Lic, L., and Ju, C. 1999. "A Study of Laser Cutting Engineering Ceramics." Optics \& Laser Technology 31: 531-8.

[9] Ji, L., Yan, Y., Bao, Y., and Jian, Y. 2009. "Laser Crack-Free Cutting Technique for Thick and Dense Ceramics." Conference on Lasers and Electro-Optics: 1-2.

[10] Lee, S. H., and Ahn, S. E. 2003. "A Probabilistic Model for Crack Formation in Laser Cutting of Ceramics." JSME International Journal Series 46 (4): 1591-7.
[11] Yan, Y., Ji, L., Bao, Y., Chen, X., and Jiang, Y. 2013. "CO2 Laser High-Speed Crack-Free Cutting of Thick-Section Alumina Based on Close-Piercing Lapping Technique." The International Journal of Advanced Manufacturing Technology 64: 1611-24.

[12] Wang, X., Zheng, H., Chu, P., Tan, J., Teh, K., Liu, T., Ang, C., and Tay, G. 2010. "Femtosecond Laser Drilling of Alumina Ceramic Substrates." Applied Physics A 101: 271-8.

[13] Lump, J., and Allen, S. 1997. "Excimer Laser Machining and Metallization of Vias in Aluminum Nitride." Transactions on Components, Packaging, and Manufacturing Technology 20 (3): 241-6.

[14] Black, I., and Chua, K. L. 1999. "Laser Cutting of Thick Ceramic Tile." Optics \& Laser Technology 4 (29): 193-205.

[15] Adelmann, B., and Hellmann, R. 2014. "Investigation on Flexural Strength Changes of Alumina Caused by Cutting Using Fiber Laser." Journal of Laser Micro/Nanoengineering 9 (2): 153-60.

[16] Liu, W., Du, W., and Liao, J. 2005. “Application of Fiber Laser Used in the Field of Stent Cutting and Micro-Machining." Lasers in Material Processing and Manufacturing II 5629: 263-70.

[17] Zhang, Z., and Modest, M. 1996. "Temperature-Dependent Absorptances of Ceramics for $\{\mathrm{Nd}: \mathrm{YAG}\}$ and $\{\mathrm{CO} 2\}$ Laser Processing Applications." In Proceedings of the Materials Processing Cutting Symposium ICALEO '96 81C: 29-38.

[18] Läpple, V. 2006. Introduction into Strength of Materials (German). Vieweg.

[19] Gross, and Seelig, T. 2006. Fracture Mechanics. Ling, F. Ed. Springer.

[20] Wee, L., Crouse, P., and Li, L. 2008. “A Statistical Analysis of Striation Formation during Laser Cutting of Ceramics." International Journal of Advanced Manufacturing Technology 36: 699-706. 